\title{
Universiteit
}

Leiden

The Netherlands

\section{Polychromatic photonic quasicrystal cavities}

Thon, S.M.; Irvine, W.T.M.; Kleckner, D.; Bouwmeester, D.

\section{Citation}

Thon, S. M., Irvine, W. T. M., Kleckner, D., \& Bouwmeester, D. (2010). Polychromatic photonic quasicrystal cavities. Physical Review Letters, 104(24), 243901.

doi:10.1103/PhysRevLett.104.243901

Version: $\quad$ Not Applicable (or Unknown)

License: $\quad$ Leiden University Non-exclusive license

Downloaded from: https://hdl.handle.net/1887/61348

Note: To cite this publication please use the final published version (if applicable). 


\title{
Polychromatic Photonic Quasicrystal Cavities
}

\author{
Susanna M. Thon, ${ }^{1, *}$ William T. M. Irvine, ${ }^{2}$ Dustin Kleckner, ${ }^{1}$ and Dirk Bouwmeester ${ }^{1,3}$ \\ ${ }^{1}$ Department of Physics, University of California Santa Barbara, Santa Barbara, California 93106, USA \\ ${ }^{2}$ Department of Physics, New York University, New York, New York 10012, USA \\ ${ }^{3}$ Huygens Laboratory, Leiden University, P.O. Box 9504, 2300 RA Leiden, The Netherlands \\ (Received 2 February 2010; revised manuscript received 14 May 2010; published 14 June 2010)
}

\begin{abstract}
Photonic crystal slabs provide unique opportunities for the manipulation of light on semiconductor chips. The patterns of holes in the slabs are typically designed to maximize the width, depth and symmetry of a single photonic band gap. Quasicrystalline patterns are ideal from this point of view; here, we show that, owing to the presence of multiple Bragg scattering length scales, they also have the desirable property of supporting multiple photonic band gaps in the same slab. This opens up the possibility of creating polychromatic cavities that could be used to extend the possibilities for single photons on optical chips, including on-chip frequency conversion in III-V semiconductors. We study several quasicrystalline structures which support high quality cavity modes at multiple resonant frequencies using 2D and 3D FDTD simulations.
\end{abstract}

DOI: 10.1103/PhysRevLett.104.243901

PACS numbers: 42.70.Qs, 42.50.Pq, 42.65.Ky

Advances in nanophotonics and photonic band gap engineering have made it possible to confine photons to onchip wavelength-scale cavities and waveguides [1-4]. This has opened the way to the realization of, among others, integrated cavity quantum electrodynamics schemes [5-7] and ultralow threshold lasers [8-10]. For these experiments, the structures were optimized to obtain the largest possible band gaps or highest quality cavity modes at a single frequency. A structure that could support multiple band gaps or cavity modes would fundamentally extend the possibilities for single photons on optical chips, for example, by allowing the exploration of on-chip nonlinear optical interactions. In this Letter, we explore photonic quasicrystals for this application. Previous work on photonic quasicrystals has concentrated primarily on the fact that they support wide, uniform band gaps due to the spherical symmetry of their Brillouin zones [11]. We show that in addition they possess the special property of supporting multiple photonic band gaps, with a small enough frequency separation that they could be supported in the same slab, because the quasiperiodicity provides two or more Bragg scattering length scales. Furthermore, unlike periodic structures, they offer a wider variety of symmetry centers for creating defect modes with different geometries [12].

An example of an application for a polychromatic cavity is on-chip frequency conversion at intensities down to the single photon level. A recent proposal [13] for observing strong coupling between single photons is illustrated in Fig. 1(a). Coherent energy transfer between a photon in cavity mode $A$ with frequency $\omega_{a}$ and a photon in a spatially overlapping cavity mode $B$ with frequency $\omega_{b}$ coupled by an optical nonlinearity can in principle be observed if a third mode with frequency $\omega_{a}-\omega_{b}$ is populated with a coherent state to make up the energy difference. The design of a cavity that can support two such overlapping modes must begin with a search for a structure that supports multiple photonic band gaps.

The formation of photonic band gaps in regular structures has been extensively studied [14]; understanding gap formation in more general structures is an active area of research $[15,16]$. The primary band gap formation mechanism is destructive interference through Bragg scattering

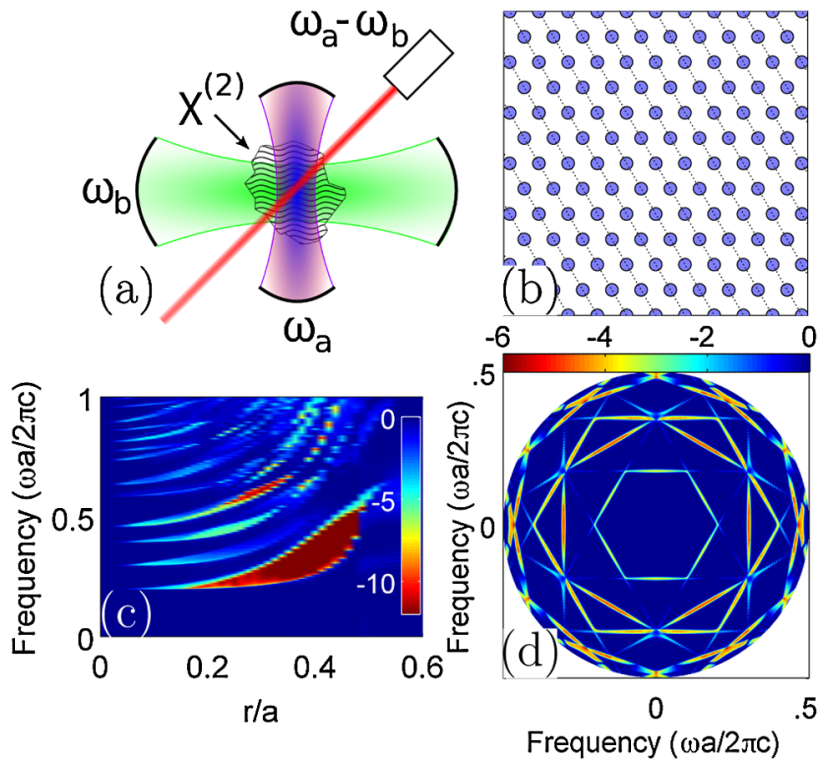

FIG. 1 (color online). (a) Schematic illustrating the requirements for frequency conversion in photonic crystal cavities. (b) Diagram of the triangular lattice. The dotted lines are the periodic Bragg plane locations associated with the $(1,1)$ direction. (c) $2 \mathrm{D} \mathrm{TE}$ band gap map at $0^{\circ}$ for the triangular lattice photonic crystal of air holes in a dielectric medium $(\epsilon=11.4)$. The color map is a logarithmic scale of normalized transmission through the lattice. (d) Angular study of the transmission structure of the triangular lattice 2D photonic crystal at $r / a=0.1$. 
$[17,18]$. Additionally, there is a Mie resonance mechanism for the formation of photonic band gaps which is only relevant for TM waves in structures where the scattering centers are formed from the higher index material [19]. The combination of these two effects was exploited in Ref. [20] to find band gaps in the different polarizations. We will concentrate on the Bragg scattering mechanism for TE waves in 2D photonic crystals based on arrays of air holes in a higher index substrate to show that photonic quasicrystal lattices of this type possess multiple band gaps at closely spaced frequencies, and could support multiple spatially overlapping modes in $2 \mathrm{D}$ and $3 \mathrm{D}$ defect structures.

We developed a transmission simulation method that makes use of the FDTD modeling technique (implemented with a commercial FDTD engine [21]), similar to that in Ref. [22], to calculate band gap maps for general, including nonperiodic, lattice structures. In our method, a broadband plane wave source is placed at one end of a finite section of the 2D photonic lattice, and the transmission is recorded in the time domain at a series of points along a line at the other end of the structure. The field components at each point are Fourier transformed and the spectra are averaged and normalized to the source and transmission profiles of a bare dielectric background to obtain the transmission as a function of frequency. A complete transmission map is generated by running separate simulations for different values of $r / a$, the ratio of the hole radius to the lattice constant (defined to be the average nearest-neighbor distance for quasiperiodic lattices). The transmission for a particular value of $r / a$ can also be studied as a function of angle by rotating the structure while keeping the source and monitor locations constant. We verified our simulation method by producing a transmission map [Fig. 1(c)] and angular study [Fig. 1(d)] of the well-characterized triangular lattice of air holes [23].

We investigated several 2D photonic quasicrystal structures using this method. The holes were placed at the vertices of tilings generated via the strip projection method [24]. In this letter, we will focus on two specific lattices: the fivefold symmetric (or Penrose) lattice generated from a five dimensional starting space, and the eightfold symmetric lattice generated from a four dimensional starting space, for which first evidence of photonic band gap formation is given in Ref. [25]. Diagrams of the fivefold and eightfold symmetric lattices are shown in Figs. 2(a) and 2(b), respectively; higher starting dimensions resulted in lattices with unrealistic fabrication parameters.

Figure 2(e) shows a band gap map calculated at zero angle of incidence for the fivefold symmetric lattice. As compared with Fig. 1(c), the transmission structure has many more dips than that of a periodic lattice, especially at low values of $r / a$. Figure 2(g) shows an angular study of the transmission structure of the same lattice plotted for $r / a=0.1$. Each of the transmission dips at small $r / a$ can be associated with Bragg scattering in a particular lattice direction. The fivefold symmetric quasicrystal lattice has

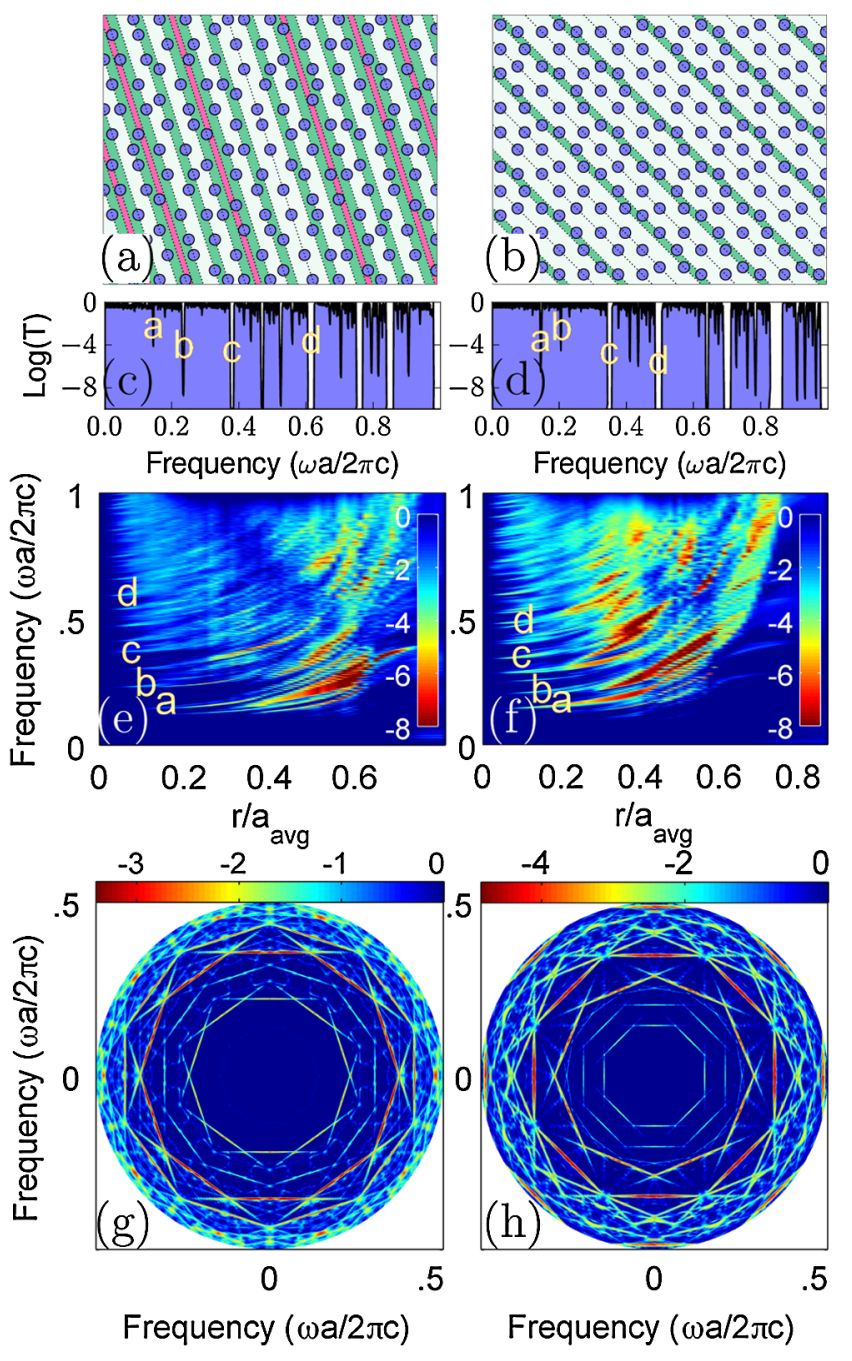

FIG. 2 (color online). (a) and (b) The 2D fivefold symmetric and eightfold symmetric lattice structures with the Bragg planes drawn in. The white sections correspond to the "Long" separation, green corresponds to the "Short" separation and pink corresponds to "Long-Short." (c) and (d) 1D simulation of quasicrystals based on the Fibonacci lattice and the eightfold symmetric quasicrystal generation rule with small scattering layers at the positions of the plane boundaries. The frequencies of the transmission dips (4 examples are labeled for comparison) correspond to those in the 2D quasicrystals at small $r / a$. (e) and (f) TE band gap maps for the fivefold symmetric and the eightfold symmetric $2 \mathrm{D}$ quasicrystal, respectively. The color map is a logarithmic scale of transmission through the lattice. $(\mathrm{g})$ and (h) Angular studies of the transmission structures of the fivefold symmetric and the eightfold symmetric $2 \mathrm{D}$ quasicrystals at small $r / a(r / a=0.1)$.

Bragg planes with spacings that correspond to a Fibonacci sequence with long $(L)$ and short $(S)$ components [see Fig. 2(a)]. The Fibonacci sequence has a generation rule $(S \rightarrow L, L \rightarrow S L)$ and characteristic equation, $\tau+1=\tau^{2}$, with a solution, $\tau$ that is equal to the golden mean, $(\sqrt{5}+1) / 2$ [26]. The ratio of the two Bragg plane spacings $(L / S)$ in the fivefold symmetric photonic quasicrystal is equal to $\tau$, and, as a result, dips in transmission are found at 
frequencies spaced by this ratio with sets of dips corresponding to different quasi-Brillouin zone lattice directions. The transmission structure at small values of $r / a$ can be confirmed by one-dimensional simulations of a Bragg mirror in which small dielectric scattering layers are interspersed with air gaps of long and short lengths arranged in the Fibonacci sequence. The result is plotted in Fig. 2(c). Transmission minima indeed appear at the same frequencies as those in the 2D fivefold symmetric photonic quasicrystal spectrum at small $r / a$ (four dips are labeled for comparison).

Because the fivefold symmetric quasicrystal lattice has two characteristic Bragg scattering scales, with a ratio of $\tau \sim 1.6$, band-gap-like features are spaced more closely together in frequency than those in periodic lattices, where complete band gap formation is possible at frequencies with approximately integer ratios: $\omega, 2 \omega, 3 \omega$, etc. In order to support two modes in the same slab photonic crystal defect structure, the frequency difference between the modes must be relatively small because the introduction of a finite thickness in the third dimension causes the closing of higher order band gaps. As discussed in Ref. [27], if the slab thickness is greater than about a wavelength in the material, then higher order vertical modes in the slab can be created which will lie only slightly above the lowest order slab guided mode, preventing the formation of an optical band gap. If the slab thickness is less than about half a wavelength in the material then the slab will provide only a weak perturbation on the background dielectric constant; any optical band gap will be minuscule. According to this criteria, the absolute largest frequency difference between separate band gaps that could be supported in the same slab would be $\omega, 2 \omega$. However, as Refs. [27,28] also discuss, the width of the supported band gap is strongly dependent on slab thickness, with the ideal thickness being closer to half a wavelength in the material, providing clear motivation to look for structures with band gaps spaced by ratios of less than $\omega, 2 \omega$.

The eightfold symmetric photonic quasicrystal transmission structure [Fig. 2(f)] has similar features as the fivefold symmetric case. There are also two characteristic lengths associated with the Bragg plane spacings, generated by a generalized Fibonacci rule $(S \rightarrow L, L \rightarrow S L L)$ which is derived from the characteristic equation $2 \tau+1=$ $\tau^{2}$, resulting in $\tau=1+\sqrt{2} \sim 2.4$. The transmission dips at small $r / a$ in Figs. 2(f) and 2(h) occur at frequencies corresponding to the lengths $L, S, L+S$, and $L-S$, and are found at two sets of spacings given by the ratios $\frac{(L+S)}{L}=\frac{(L-S)}{S}=\tau-1=\sqrt{2} \sim 1.4$, and $\frac{L}{(L-S)}=\frac{\tau}{(\tau-1)}=$ $1+\frac{1}{\sqrt{2}} \sim 1.7$.

We investigated several defect structures in the previously studied 2D eightfold symmetric photonic quasicrystal lattice [29]. We will describe the results for the defect cavity shown in Fig. 3(c). FDTD cavity ring down simulations were performed at $r / a=0.375$ which was chosen
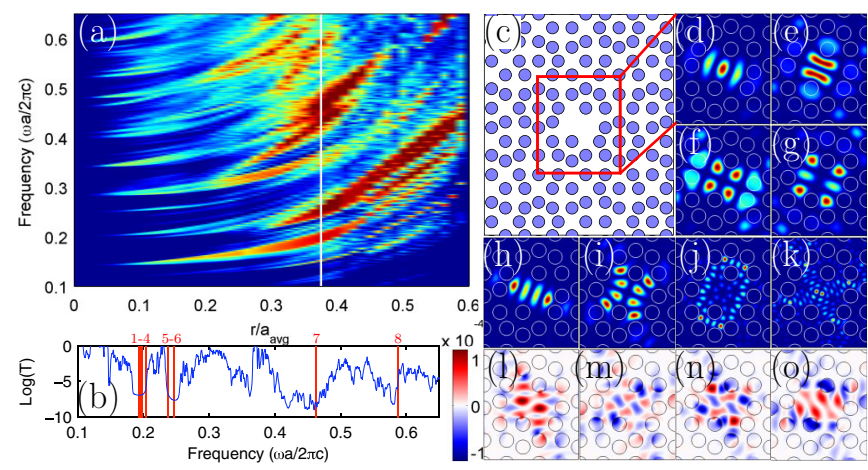

FIG. 3 (color online). (a) Band gap map for the eightfold symmetric photonic quasicrystal. The white line indicates the parameters used for the 2D mode simulation $\left(r / a_{\text {avg }}=0.375\right)$. (b) $1 \mathrm{D}$ slice of the transmission $(T)$ spectrum at $r / a_{\text {avg }}=0.375$ with the positions of the eight confined modes marked. (c) Diagram of the cavity defect structure. (d)-(k) Mode intensity (arbitrary units) plots of the $H_{z}$ field component for eight confined modes. Simulated quality factors: (d) Mode 1: $Q=$ $2 \times 10^{8}$ (e) Mode 2: $Q>5 \times 10^{7}$ (f) Mode 3: $Q=2 \times 10^{8}$ (g) Mode 4: $Q=1 \times 10^{7}$ (h) Mode 5: $Q>10^{8}$ (i) Mode 6: $Q>$ $10^{8}$ (j) Mode 7: $Q=1 \times 10^{6}$ (k) Mode 8: $Q=6 \times 10^{4}(\mathrm{l})$ (o) Plot of the overlap of the different field components of modes 3 and 6. (l) $E_{x 3} * E_{x 6}$, (m) $E_{x 3} * E_{y 6}$, (n) $E_{y 3} * E_{x 6}$, (o) $E_{y 3} * E_{y 6}$.

as a compromise between the presence of strong transmission minima and the practicality of fabrication. The mode intensity for a set of strongly confined modes is shown in Figs. 3(d)-3(k) along with their associated 2D quality factors. We can locate each of the modes in a transmission minima as shown in Fig. 3(b). In order to evaluate the modes based on their suitability for the frequency conversion scheme, we calculated the spatial overlap of pairs of modes located in different transmission minima features. The tensorial nature of the $\chi^{(2)}$ interaction must be taken into account by contracting the electric field vectors of the cavity modes with the $\chi^{(2)}$ tensor. In $\mathrm{GaAs}$, the lattice can be oriented, for example, such that the polarizations of the two cavity modes and the weakly confined third mode are aligned (TE) and the growth direction is (111) [13]. The spatial overlap was calculated in $2 \mathrm{D}$ by integrating the products of the field components separately over the $x-y$ plane, normalized such that

$$
\int\left(E_{x n} E_{x n}+E_{y n} E_{y n}\right) d x d y=1
$$

A value of 0.5 for any pair of components would indicate maximum spatial overlap. Figures 3(1)-3(o) contain plots of the products of the field components of modes 3 [Fig. 3(f)] and 6 [Fig. 3(i)]. The overlap of these mode components ranges from 0.04 to 0.12 , indicating an overlap of $8 \%-24 \%$ of the maximum possible, which could lead to a significant improvement in conversion efficiency over previous schemes that used only one optimized cavity mode $[30,31]$. 
3D FDTD simulations indicate that quality factors of at least $10^{3}$ can be maintained in pairs of modes in separate band gaps after the introduction of a finite slab thickness. The level of overlap of the two cavity mode components can only marginally differ in $3 \mathrm{D}$ from its $2 \mathrm{D}$ value because both modes display first order modal character in the third dimension for any slab thickness which will support both band gaps, as confirmed by 3D simulations. This allows us to apply our results from 2D systems directly to the design of 3D structures. There is a corresponding frequency shift associated with the move to $3 \mathrm{D}$ that tends to reduce the ratio of the mode frequencies (for instance, modes 2 and 5 can be maintained with a frequency ratio of 1.16 and quality factors of $10^{3}$ in the same slab, compared to a ratio of 1.20 in 2D). Operation in the strong coupling regime defined in Ref. [13] requires that the effective Rabi oscillation period be greater than the effective photon cavity lifetime. The Rabi oscillation frequency is given by [13]

$$
\hbar \Omega=\epsilon_{0}\left(\frac{\hbar}{2 \epsilon_{0}}\right)^{3 / 2} \sqrt{\frac{\omega_{a} \omega_{b} \omega_{c}}{n_{a}^{2} n_{b}^{2} n_{c}^{2} V_{a} V_{b} V_{c}}} \int d V \chi_{i j k}^{(2)} E_{a}^{i} E_{b}^{j} E_{c}^{k},
$$

where $\chi_{i j k}^{(2)}$ is the nonlinear susceptibility tensor, $E_{a, b, c}$ and $V_{a, b, c}$ represent the spatial parts of the modes $a, b, c$ normalized such that their maximum value is 1 , and the mode volumes, respectively. Making the same assumptions as those given in Ref. [13] ( $V_{c}=f_{c} V_{a}, f_{c}$ is 1-100, $\lambda_{b} \sim$ $1.5 \mu \mathrm{m}$ ), the Rabi oscillation period depends only on the number of photons in the third (coherent state) mode, and the effective photon lifetime can be calculated from the cavity quality factors. This leads to the estimate that the strong coupling regime could be reached in our cavities with an average of $10^{9}$ photons in the third mode, populated by a weakly confined laser beam. In this case, the conversion efficiency for the photons at the frequency of interest (i.e., those in mode a) is given by $\eta_{a b}=\gamma_{b} /\left(\gamma_{a}+\gamma_{b}\right)$, where $\eta_{a b}$ is the efficiency with which photons in mode a are converted to photons in mode $b$, and $\gamma_{a, b}$ is the decay rate of cavity $a, b$.

In conclusion, we have demonstrated that photonic crystals based on quasiperiodic lattices can support multiple band gaps at frequencies more closely spaced than those in periodic lattices due to the presence of multiple Bragg scattering length scales. By taking advantage of this unique structure, we were able to identify defect cavities in the eightfold symmetric quasicrystal lattice that possess multiple spatially overlapping modes, and apply our 2D results to the design of full 3D slab structures. We expect that this design can be further optimized by fine-tuning the hole positions and sizes near the defect [32]. Once fabricated in a highly nonlinear material such as GaAs or GaP, these cavities are promising candidates for achieving efficient on-chip frequency conversion.
This work was supported by NSF Grant No. 0901886 and Marie Curie EXT-CT-2006-042580. S. M. T. acknowledges financial support from a U.S. Department of Education GAANN grant. W. T. M. I. acknowledges support from the English Speaking Union.

*susanna@physics.ucsb.edu

[1] J. P. Reithmaier et al., Nature (London) 432, 197 (2004).

[2] M. T. Rakher et al., Phys. Rev. Lett. 102, 097403 (2009).

[3] K. Srinivasan and O. Painter, Nature (London) 450, 862 (2007).

[4] C. Santori et al., Nature (London) 419, 594 (2002).

[5] D. Englund et al., Nature (London) 450, 857 (2007).

[6] T. Yoshie et al., Nature (London) 432, 200 (2004).

[7] K. Hennessy et al., Nature (London) 445, 896 (2007).

[8] J. Wiersig et al., Nature (London) 460, 245 (2009).

[9] S. Strauf et al., Phys. Rev. Lett. 96, 127404 (2006).

[10] A. Strittmatter et al., Appl. Phys. Lett. 88, 262104 (2006).

[11] W. Man et al., Nature (London) 436, 993 (2005).

[12] S. Cheng et al., Phys. Rev. B 59, 4091 (1999).

[13] W. T. M. Irvine, K. Hennessy, and D. Bouwmeester, Phys. Rev. Lett. 96, 057405 (2006).

[14] S. G. Johnson and J. D. Joannopoulos, Opt. Express 8, 173 (2001).

[15] M.C. Rechtsman and S. Torquato, Phys. Rev. B 80, 155126 (2009).

[16] M. Florescu, S. Torquato, and P. J. Steinhardt, Phys. Rev. B 80, 155112 (2009).

[17] W. Steurer and D. Sutter-Widmer, J. Phys. D 40, R229 (2007).

[18] C. Rockstuhl, U. Peschel, and F. Lederer, Opt. Lett. 31, 1741 (2006)

[19] L. Shi, X. Jiang, and C. Li, J. Phys. Condens. Matter 19, 176214 (2007).

[20] L. C. Andreani and D. Gerace, Phys. Rev. B 73, 235114 (2006).

[21] Simulations were performed using the Lumerical FDTD Solutions software package.

[22] R. Gauthier and K. Mnaymneh, Opt. Express 13, 1985 (2005).

[23] J. Joannopoulos, R. Meade, and J. Winn, Photonic Crystals Molding the Flow of Light (Princeton University Press, Princeton, New Jersey, 1995), p. 125.

[24] U. Vogg and P. L. Ryder, J. Non-Cryst. Solids 194, 135 (1996).

[25] Y. S. Chan, C. T. Chan, and Z. Y. Liu, Phys. Rev. Lett. 80, 956 (1998).

[26] D. Levine and P. J. Steinhardt, Phys. Rev. B 34, 596 (1986).

[27] S. G. Johnson et al., Phys. Rev. B 60, 5751 (1999).

[28] L. C. Andreani and M. Agio, IEEE J. Quantum Electron. 38, 891 (2002).

[29] D. M. Beggs, M. A. Kaliteevski, and R. A. Abram, J. Mod. Opt. 54, 881 (2007).

[30] K. Rivoire et al., Opt. Express 17, 22609 (2009).

[31] M. W. McCutcheon et al., Appl. Phys. Lett. 95, 221102 (2009).

[32] Y. Akahane et al., Nature (London) 425, 944 (2003). 